\title{
Acute pancreatitis and diabetic ketoacidosis in pregnancy: a case report
}

\author{
Ethayakumar N, Ragunathan MK \\ Teaching Hospital, Karapitiya, Galle \\ Corresponding author; Dr. N. Ethayakumar (drethayam@gmail.com)
}

\section{Introduction}

Acute pancreatitis leading to diabetic ketoacidosis in pregnancy is an extremely rare but serious event. The most common causative factor is severe hypertriglyceridaemia. Early detection of such life threatening situation is very important to minimize both maternal and foetal morbidity. Here we report such a case with normotriglyceridaemia.

\section{Case Report}

A 30-year-old woman in her second pregnancy was admitted to our medical unit with the complaints of chest tightness, breathlessness, thirst and epigastric discomfort of one day, at 35 weeks of gestation. There was no history of fever or recent weight loss. There was no past medical history of bronchial asthma, cardiac disorder, diabetes mellitus or hypertension. She made regular antenatal visits where there was no documented evidence of impaired glucose tolerance or diabetes. She had no family history of diabetes mellitus or lipid disorders. She was on vitamin supplements only. She had not taken Ayurvedic treatment or misused alcohol. Her first pregnancy was terminated at 38 weeks of gestation with caesarian section due to major degree of placenta praevia and delivery was uneventful.

On examination she was averagely built, tachypnoeic with notable acidotic breathing and mildly dehydrated. She was afebrile and not pale. Her pulse rate was 110 per minute and regular, and her blood pressure was 100/70 mm Hg. Her oxygen saturation at room air was $98 \%$. There was moderate epigastric tenderness and the fundal height was compatible with her gestational age. Foetal heart rate was about 140 beats/min. Rest of the examination was unremarkable.

Her capillary blood sugar was more than $500 \mathrm{mg} / \mathrm{dL}$ on admission, arterial blood gas revealed metabolic acidosis with the $\mathrm{pH}$ of 7.1. Her urine was positive for ketone bodies. Her full blood count showed mild leucocytosis $\left(11.3 \times 10^{9} / \mathrm{L}\right)$ with normal differential count. Blood urea and serum creatinine were within the normal range. She was considered having diabetic ketoacidosis and treatment was started appropriately. She needed more than 150 units of short acting insulin during the first 24 hours and the insulin requirement went down gradually. Abdominal ultrasonography showed an oedematous pancreas with hypoechogenicity compatible with acute pancreatitis without evident biliary tract disease or pancreatic calculus. Serum amylase level initially was $1640 \mathrm{U} / \mathrm{L}$. Her lipid profile was within the normal limits.

She was managed in the intensive care unit appropriately for 1 week, and due to foetal distress, emergency caesarian section was performed and a normal baby boy was delivered. Her serum amylase reduced to $264 \mathrm{U} / \mathrm{L}$ within a week. She was discharged on the tenth day on human biphasic 30/70 insulin twice daily.

\section{Discussion}

Acute pancreatitis in pregnancy is a rare but serious event and is seen in approximately 3 in 10,000 births. It is more common with advanced gestational age and seen mostly in the third trimester (1). The major causes for acute pancreatitis in pregnancy include biliary tract diseases especially related to gallstones (account for 70\%) and hypertriglyceridaemia (2). Hypertriglyceridaemia could be due to pregnancy itself as it causes 2-4 fold rise in triglyceride more pronounced in the third trimester (3), or due to diabetes which also causes deranged lipoprotein metabolism and gives rise to hypertriglyceridaemia. It would be more prominent if the patient is a preexisting hypertriglyceridaemic.

Acute pancreatitis and diabetic ketoacidosis in pregnancy is extremely rare, and is mainly due to hypertriglyceridaemia. But in this patient both above 
mentioned aetiological factors were excluded. Cases of acute pancreatitis with diabetic ketoacidosis have been reported with the use of some antipsychotic drugs such as olanzapine (4), but this patient denied use of such medications.

Severe diabetic ketoacidosis and hyperglycaemia with associated dehydration are known risk factors of acute pancreatitis (5). On admission, this patient had a low $\mathrm{pH}$ and high capillary blood sugar which probably were the risk factors in her case.

Failure to offer timely, appropriate management in this setting will result in an unfavorable outcome to mother and foetus. Treatment delay could lead to acute renal failure and disseminated intravascular coagulation in the mother and loss of the foetus. Prompt and accurate diagnosis with appropriate management resulted in a good outcome for the mother and the foetus in our case.

\section{References}

1. Pitchumoni CS, Vegneswaran B. Acute pancreatitis in pregnancy. World J Gastroenterol 2009; 15(45): 5641-6.

2. Ramin KD, Ramin SM. Acute pancreatitis in pregnancy. Am JObstet Gynaecol 1995; 173: 187-191.

3. Shih-Yi Lin, Ya-Yu Wang. Gestational pancreatitis complicating uncontrolled diabetes mellitus. Postgrad Med J 1999; 75: 241-3.

4. Khan D, Bourgeois JA. Acute pancreatitis and diabetic ketoacidosis in a patient taking olanzapine. $J$ Clin Psychopharmacol 2007; 27(4):397-400.

5. Shigetazu N, Masutoshi M. Acute pancreatitis with diabetic ketoacidosis associated with hypermyoglobinaemia. J Gastroenterol 1996.

\title{
Dengue haemorrhagic fever presenting as acute acalculous cholecystitis: a case report
}

\author{
Dissanayake DRRHB, Fernando A, Lekamwasam JDVC \\ Teaching Hospital, Karapitiya, Galle. \\ Corresponding author; Dr. D.R.R.H.B.Dissanayake (ravidissanayake@gmail.com)
}

\section{Introduction}

Dengue fever is estimated to affect 100 million people annually worldwide, usually presents as a mild febrile illness and less frequently as a haemorrhagic fever with shock (1). Unusual clinical presentations that mimic other common emergencies can, however, occasionally occur (2). A high clinical suspicion is required to make an early diagnosis and initiate prompt treatment. If unrecognized, delay in treatment can lead to disastrous outcomes. Here we present an unusual clinical presentation of dengue fever mimicking a common surgical and gastroenterological emergency.

\section{Case Report}

A 49-year old female presented with a 4-day febrile illness associated with epigastric discomfort radiating to the right hypochondrium. The pain was dull and intermittent but increasing in intensity.
This was associated with generalized myalgia and weakness. She also complained of bleeding per vagina for two days.

She had no significant medical or travel history. At presentation she was tachycardiac, with a pulse rate of $100 / \mathrm{min}$ and a blood pressure of $90 / 60 \mathrm{mmHg}$. Clinically she was flushed and mildly dehydrated. Tenderness with guarding was elicited in the right hypochondrium, with a positive Murphy's sign. Rest of the abdomen was mildly distended but was soft. There was no skin rash and the systemic examination was normal. The clinical diagnosis of acute cholecystitis with septic shock was made.

The patient was resuscitated with intravenous crystalloids and started on intravenous ciprofloxacin therapy. Abdominal ultrasound scan confirmed the presence of a thickened gallbladder wall with a positive ultrasound Murphy's sign but there were no gallstones. No ascites was evident. 